\section{A study of paradoxical sleep depth in the rat*}

\author{
KENNETH DAVIS, ELIZABETH HEINS, and HENRY VAN TWYVER \\ Florida Technological University, Orlando, Fla. 32816
}

Arousal tests were conducted on five rats implanted with EEG electrodes to monitor sleep stages. The arousing stimulus was mild vibration of the cage floor, which by several criteria can be considered a biologically relevant stimulus. Behavioral and EEG reactions to this stimulus were compared for wakefulness, paradoxical sleep, and slow-wave sleep. Probability of arousal was high during wakefulness and slow-wave sleep, but much lower during paradoxical sleep. This finding has relevance for one interpretation of the "sentinel" hypothesis of paradoxical sleep. It indicates paradoxical sleep to be a deeper phase than slow-wave sleep.

The mammalian sleep pattern consists of two distinct phases: a stage of slow-wave sleep with high-voltage low-frequency waves (SWS) and a stage of low-voltage fast or paradoxical sleep (PS). Paradoxical sleep might have evolved phylogenetically as a protective mechanism to allow an animal to monitor its environment for danger while sleep continues. This is an extension of the "sentinel" hypothesis which in its strictest form refers to the arousal periods which sometimes follow PS (Snyder, 1966, 1969). If PS serves a "sentinel" function, it should be a lighter stage of sleep. Thus, it would be of interest to know the relative depth of each of these phases. One way that this can be determined is by measuring the intensity of stimulation necessary to arouse the sleeping animal. Using this technique, it has been shown that neutral stimuli such as auditory signals or electrical shocks to the neck muscles or reticular formation result in fewer arousals during PS than during SWS (see references in Van Twyver \& Allison, 1970). Similarly, if a tone which has acquired "meaning" by paired presentations with electric shocks is used to elicit arousal, PS is a less sensitive state than SWS (Buendia et al, 1963; Van Twyver \& Garrett ${ }^{1}$ ). Thus, for learned meaningful stimuli and for neutral stimuli, arousal is more difficult to elicit from PS than from SWS, and it appears doubtful that PS could serve a "sentinel" function. Before a "sentinel" function can be completely ruled out, however, tests using stimuli which have innate arousing capacities must be conducted. In the present study, a vibratory stimulus was employed to study depth of sleep. Previous observations had indicated that vibration was a very reliable stimulus for itilucing brief arousals. Unlike auditory stimuli or neck-muscle shocks, there appeared to

*Supported by PHS Grant No. NIMH $1 \mathrm{RO} 3 \mathrm{MH} 20 \mathrm{H} 6 \mathrm{H}-01$. be little or no tendency for habituation of the arousal response, even after many presentations. Thus, on this basis at least, it could be argued that for the rat, vibration has innate meaning as an arousing stimulus.

\section{METHODS}

Five young adult albino rats were surgically prepared with six surface screw electrodes for recording electroencephalograms (EEGs) and two loops of stainless steel wires into the neck for electromyogram (EMG) recording. The anesthesia was $45 \mathrm{mg} / \mathrm{kg}$ sodium pentobarbital. Following a recovery period, arousal tests were carried out in a During arousal tests, EEGs were recorded by means of a Gilson M8PM polygraph with simultaneous visual observations. Frequency response of EEG channels was flat from 0.8 to $20 \mathrm{~Hz}$. The vibratory stimulus was produced by a dc electric motor with an eccentrically counterweighted shaft connected to the cage floor by direct mechanical linkage. Intensity of vibration was controlled by regulating the voltage of the power unit supplying the motor. During preliminary tests, stimulus intensity was set at a value which reliably produced arousals during SWS. This value, determined with a General Radio sound-pressure-level meter, was $5 \mathrm{~dB}$ (re: .0002 microbar; ambient noise level, $56 \mathrm{~dB}$ ). The meter was one longitudinal floor support. Measurement by means of a stylus attached to the floor support indicated 0.5 to $1.0 \mathrm{~mm}$ maximum excursion. This value was held constant throughout the course of arousal testing. Each rat was given from 40 to 505 -sec presentations of the vibratory stimulus during wakefulness (W) (low-amplitude high-frequency EEG, with high or moderate EMG), SWS (high-amplitude low-frequency EEG, with moderate or low EMG), and PS sound-deadening recording chamber. placed at a $90 \cdot \mathrm{deg}$ angle $5 \mathrm{~mm}$ from (low-amplitude high-frequency EEG with minimal EMG). Arousal tests were separated by at least $1 \mathrm{~min}$ but usually by a much longer interval. This was because several minutes were usually required for sleep to resume following arousal. Trials were presented at a fairly equal rate in each of the three states to reduce the possibility that decreased responding could affect one state more than another.

\section{RESULTS}

During $W$, arousals consisted of an orienting movement of the head followed by a quiet period. If the stimulus came on during an active period, activity would usually cease for a short time, with the head held erect. Nonarousals during $\mathrm{W}$ consisted of a failure to orient during the stimulus presentation. SWS arousals were indicated by EEG desynchronization accompanied by an orienting movement. On some trials, temporary EEG desynchronization unaccompanied by an orienting movement was seen; such trials were scored as nonarousals. During PS, arousals consisted primarily of the orienting movement. EEG patterns were of minimal value in determining PS arousals because it is difficult to detect the relatively slight differences between waking EEG patterns and the desynchronized EEG of PS. Stimulus presentations which did not produce a full arousal sometimes were followed by a shift to the SWS pattern. This is a common finding (cf. Van Twyver \& Allison, 1970). These were not counted as arousals in the present study.

Probability of arousal was lowest during $P S$, and rather high during SWS and $\mathrm{W}$ for each rat (Fig. 1). This result is in complete agreement with the previous studies of sleep depth cited in the introduction section. In all cases, overall probability of behavioral arousal was at least as great during SWS as during $W$. In four of five rats, the overall probability was even higher during SWS than during W. This was due to the high level of efficiency of vibration as an arousing stimulus and to the absence of response habituation during sleep (Fig. 2). Initially, there was an equally high probability of arousal during $W$ and SWS. Responding decreased slightly across trial blocks for W but not for SWS. This decrease was responsible for the slightly lower arousal probabilities during $W$.

Vibration may have innate arousing capacities for the rat. First, it does not seem to lose its capacity to elicit arousal, even after many trials. In contrast, neutral stimuli do not reliably elicit arousal after several 
presentations (Dillon \& Webb, 1965; Van Twyver \& Garrett ${ }^{1}$ ). Second, vibration produced arousal during SWS as efficiently as it did during $W$. This is not true of neutral stimuli or stimuli which have attained meaning through avoidance conditioning (Van Twyver \& Allison, 1970; Van Twyver \& Garrett ${ }^{1}$ ).

A third line of reasoning, more intuitive than empirical, supports our contention that vibration has innate arousing capacities. In nature, vibration of the nest or burrow may serve as a danger signal. It would be biologically adaptive for any species which sleeps deeply to awaken to movement of its nest. Presumably, a predator large enough to be a threat would cause movement something like the stimulus used in our arousal tests.

It is clear that for the stimulus and subject employed and for our criterion of sleep depth, PS is a deeper stage of sleep than SWS. This result is made more striking by the high level of reactivity which was seen during SWS and is difficult to reconcile with a theory that postulates PS as a sentinel mechanism. Rather than having biological utility, in this context at least, PS is a nonadaptive state. The weight of evidence therefore presently favors the conclusion that PS itself is not a stage of sleep beneficial for monitoring the external environment. It is possible that the domestic rat has lost the ability to react during PS in the way that his earlier forebears might have done, but this seems unlikely, because other PS characteristics are essentially the same in wild and domesticated species (Allison \& Van Twyver, 1970).

Species which are constantly exposed to predators (most notably the ruminants) have very low percentages of PS compared to those which customarily sleep in a secure den or burrow (Allison \& Van Twyver, 1970). This reduction would now seem to be a specialization due to the lack of sensitivity to environmental events which occurs during PS. In those species constantly exposed to predation, there would be biological pressure for reduction of PS.

It is possible that the brief arousals which sometimes conclude PS periods might serve to interrupt sleep in order

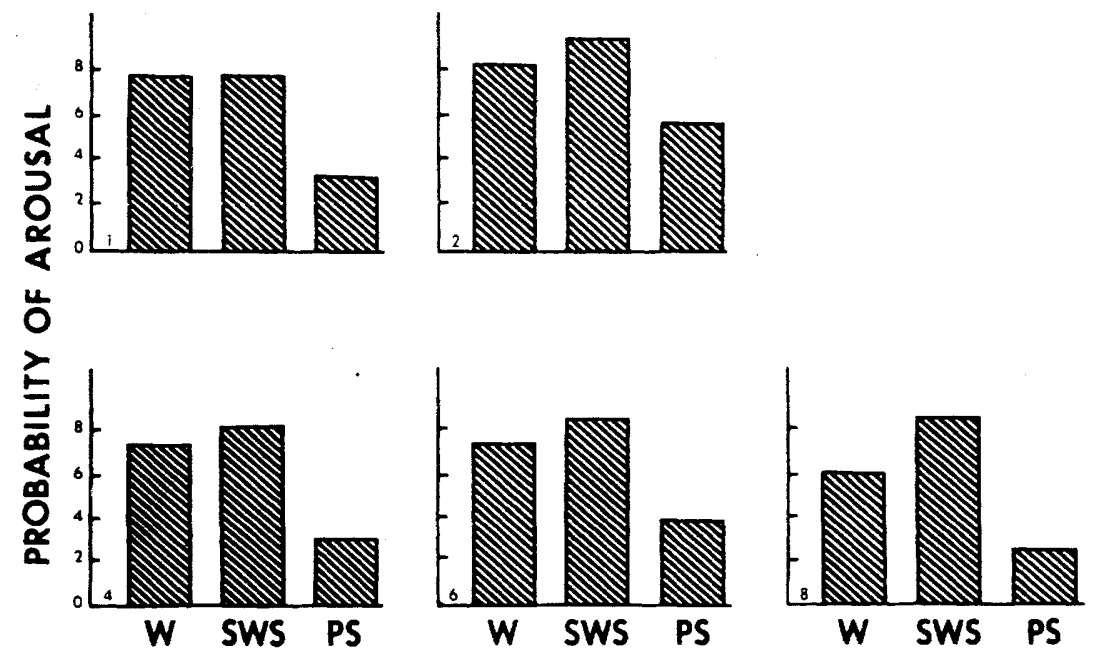

Fig. 1. Probability of eliciting EEG and behavioral arousal with a vibratory stimulus during $W$, SWS, and PS. In all cases arousal frequency was lowest during PS. High frequency of arousal during SWS and tendency to not habituate during SWS (see Fig. 2) indicate vibration is an efficient stimulus.

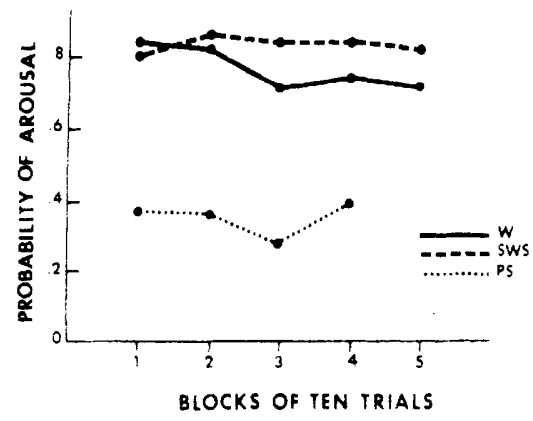

Fig. 2. Probability of arousal across trial blocks (means for five rats). There was a slight tendency for habituation of arousal during wakefulness, but no habituation during SWS or PS. Several rats did not complete 50 trials during PS, so this data point has been omitted.

to provide an opportunity for an animal to monitor its environment (Snyder, 1969). Hence, the importance of PS may be in these brief arousal periods rather than in the PS periods themselves, a possibility that remains to be investigated.

\section{REFERENCES}

ALLISON, $T$, \& VAN TWYVER, $H$. The evolution of sleep. Natural History, 1970 79, 56-65

BEUNDIA, N., SIERRA, G., GOODE, M., \& SEGUNDO, J. Conditioned and discriminatory responses in wakeful and in sleeping cats. Electroencephalography Supplement 24, 199-218.

DILLON, R. F., \& WEBB, W. B. Threshold of arousal from 'activated' sleep in the rat. Journal of Comparative \& Physiological Psychology, 1965, 59, 446-447.

SNYDER, F. Toward an evolutionary theory of dreaming. American Journal of Psychiatry, 1966, 123, 121-136.

SNYDER, F. Sleep and REM as biological enigmas. In A. Kales (Ed.), Sleep: Physiology and pathology. Philadelphia: Lippincott, 1969.

VAN TWYVER, H., \& ALLISON, T. Sleep in the opossum, Didelphis marsupialis, Electroencephalography \& Clinical Neurophysiology, 1970, 29, 181-189.

\section{NOTE}

1. Van Twyver, H., \& Garrett, W. Arousal threshold in the rat determined by "meaningful" stimuli. In preparation. 